\title{
Theoretical Evaluation of Ultrasonic Velocities in Ternary Liquid Mixture of Tetrahydrofuran, Heptane and Decane at Different Temperatures
}

\author{
S. BALAKRISHNAN \\ Department of Physics, Achariya College of Engineering Technology, Puducherry, India \\ bala.phy@gmail.com
}

Received 6 October 2014 / Accepted 17 October 2014

\begin{abstract}
Ultrasonic velocities and densities of the ternary liquid mixtures of tetrahydrofuran, heptanes and decane have been measured at temperatures $303 \mathrm{~K}, 308 \mathrm{~K}$ and $313 \mathrm{~K}$ over the entire composition range of mole fractions. The theoretical values of ultrasonic velocity were evaluated using Nomoto's Relation $\left(U_{N R}\right)$, Junjie's Method $\left(U_{J M}\right)$, Ideal Mixing Relation $\left(U_{I M R}\right)$, Free Length Theory $\left(\mathrm{U}_{\mathrm{FLT}}\right)$, Collision Factor Theory $\left(\mathrm{U}_{\mathrm{CFT}}\right)$ and Impedance Dependence Relation $\left(\mathrm{U}_{\mathrm{IDR}}\right)$. Theoretical values are compared with the experimental values and the validity of the theories is checked by calculating the average percentage error (APE).
\end{abstract}

Keywords: Ultrasonic velocity, Collision factor theory, Ternary liquid mixtures, Average Percentage Error

\section{Introduction}

During the last two decades there has been an increasing interest in the study of intermolecular interactions and numbers of experimental techniques have been used to investigate the interactions between the ternary liquid mixture components. Ultrasonic study of liquids, due to its nondestructive nature, has been extensively carried out in different branches of science to measure the thermodynamic properties and to predict the nature of molecular interactions between the molecules in a medium ${ }^{1}$. The ultrasonic velocity and the thermodynamic parameters derived from it have been widely used to interpret the interactions between unlike molecules in the ternary liquid mixtures. Several researchers ${ }^{2-5}$ carried out ultrasonic investigations and correlated the experimental results of ultrasonic velocity with the theoretical relations of Nomoto $^{6}$, Junjie ${ }^{7}$, Van Deal and Vangeel $^{8}$, Jacobson ${ }^{9}$, Schaaf ${ }^{10}$ and Impedance dependence relation $^{11}$. In the present communication the aforementioned relations have been used to predict ultrasonic velocity in ternary liquid mixture of tetrahydrofuran with heptane and decane.

\section{Experimental}

All the chemical used are of analytical reagent (AR) and spectroscopic reagent (SR) without further purification. The purities of the above chemicals were checked by density determination 
at 303, 308 and $313 \pm 0.1 \mathrm{~K}$, which showed an accuracy of $\pm 1 \times 10^{-4} \mathrm{gcm}^{-3}$. The ternary liquid mixtures of different known compositions were prepared in stopper measuring flasks. The density and velocity were measured as a function of composition of the ternary liquid mixture at 303, 308 and $313 \mathrm{~K}$ for mixed solvent systems in which tetrahydrofuran was added to a binary mixtures of heptane and decane. For this purpose binaries with fixed volume ratios $\mathrm{X}_{2} / \cong 3: 1$ were prepared. The density was determined using a specific gravity bottle by relative measurement method with an accuracy of $\pm 0.01 \mathrm{kgm}^{-3}$. The weight of the sample was measured using electronic digital balance with an accuracy of $\pm 0.1 \mathrm{mg}$ (Model: Shimadzu AX-200). An ultrasonic interferometer having the frequency of $3 \mathrm{MHz}$ (Mittal Enterprises, New Delhi, Model: F-81) with an overall accuracy of $\pm 0.1 \%$ has been used for velocity measurement. An electronically digital operated constant temperature bath (RAAGA Industries) has been used to circulate water through the double walled measuring cell made up of steel containing the experimental solution at the desired temperature. The accuracy in the temperature measurement is $\pm 0.1 \mathrm{~K}$.

\section{Theory and Calculations}

Nomoto's Relations (NR)

$$
U_{\text {NOM }}=\left[\frac{X_{1} R_{1}+X_{2} R_{2}+X_{3} R_{3}}{X_{1} V_{1}+X_{2} V_{2}+X_{3} V_{3}}\right]^{3}
$$

Where, Molar sound velocity,

$$
R_{1}=\frac{m_{1}}{d_{1}} U_{1}^{1 / 3} ; R_{2}=\frac{m_{2}}{d_{2}} U_{2}^{1 / 3} ; R_{3}=\frac{m_{3}}{d_{3}} U_{3}^{1 / 3}
$$

Molar volume $V_{1}=\frac{m_{1}}{d_{1}} ; V_{2}=\frac{m_{2}}{d_{2}} ; V_{3}=\frac{m_{3}}{d_{3}}$

Junjie's Method (JM)

$U_{J M}=\left[\frac{X_{1} V_{1}+X_{2} V_{2}+X_{3} V_{3}}{X_{1} m_{1}+X_{2} m_{2}+X_{3} m_{3}}\right]^{1 / 2}\left[\frac{X_{1} V_{1}}{d_{1} U_{1}^{2}}+\frac{X_{2} V_{2}}{d_{2} U_{2}^{2}}+\frac{X_{3} V_{3}}{d_{3} U_{3}^{2}}\right]^{-1 / 2}$

Ideal Mixing Relation (IMR)

$$
U_{I M R}=\left[\frac{1}{X_{1} m_{1}+X_{2} m_{2}+X_{3} m_{3}}\right]^{1 / 2}\left[\frac{X_{1}}{m_{1} U_{1}^{2}}+\frac{X_{2}}{m_{2} U_{2}^{2}}+\frac{X_{3}}{m_{3} U_{3}^{2}}\right]^{-1 / 2}
$$

The degree of molecular interaction $(\alpha)^{12}$ is given by

$\alpha=\frac{U_{\text {exp }}^{2}}{U_{\text {IMR }}^{2}}-1$

Free Length Theory (FLT)

$$
U_{C F T}=\frac{K}{L_{f_{\text {mix }}} d_{\exp }^{1 / 2}}
$$


Where, $L_{\text {fmix }}=2\left[\frac{V_{m}-\left(X_{1} V_{01}+X_{2} V_{02}+X_{3} V_{03}\right)}{X_{1} Y_{1}+X_{2} Y_{2}+X_{3} Y_{3}}\right]$

Molar volume at absolute zero, $V_{o 1}=V_{1} \frac{U_{1}}{U_{\infty}} ; V_{02}=V_{2} \frac{U_{2}}{U_{\infty}} ; V_{03}=V_{3} \frac{U_{3}}{U_{\infty}}$

Surface area per mole, $Y_{1}=\frac{2\left(V_{1}-V_{01}\right)}{L_{f_{1}}} ; Y_{2}=\frac{2\left(V_{2}-V_{02}\right)}{L_{f_{2}}} ; Y_{3}=\frac{2\left(V_{3}-V_{03}\right)}{L_{f_{3}}}$

Where, 1, 2, represents the first and second component of the liquid mixture and the other symbols have their usual meanings.

Impedance dependence relation (IDR)

$U_{I D R}=\frac{X_{1} Z_{1}+X_{2} Z_{2}+X_{3} Z_{3}}{X_{1} \rho_{1}+X_{2} \rho_{2}+X_{3} \rho_{3}}$

Collision Factor Theory (CFT)

$U_{C F T}=\frac{U \infty \sum x_{i} S_{i} \sum x_{i} B_{i}}{V_{\text {mix }}}$

Here, $\mathrm{U}_{\propto}=1600 \mathrm{~ms}^{-1}$, $\mathrm{S}$ is the collision factor and $\mathrm{B}$ the actual volume of molecule per mole, given as,

$B=\frac{4 \pi}{3} r_{m N}^{3}$

Where $r_{m}$ stands for molecular radius and $\mathrm{N}$ the Avogadro number. The value of $r_{m}$ has been obtained using Schaaffs expression as follows

$r_{m}=\left(\frac{3 b}{16 \pi N}\right)^{1 / 3}$
$b=\left(\frac{m}{p}\right)\left[1-\frac{R T}{m u^{2}}\left\{\sqrt{1+\frac{m U^{2}}{3 R T}-1}\right\}\right]$

Average percentage error $(A P E)^{13}$

$A P E=\frac{1}{n} \sum_{i=1}^{n} \frac{U_{\text {exp }}-U_{\text {theo }}}{U_{\exp }} \times 100$

Where, n- number of data used.

$\mathrm{U}_{\text {exp }}=$ experimental values of ultrasonic velocities of mixtures.

$\mathrm{U}_{\text {theo }}=$ theoretically computed values of ultrasonic velocities of mixtures.

In all the above equations the symbols used have their usual meanings.

\section{Results and Discussion}

The values of ultrasonic velocities computed theoretically using the relations of Nomoto, Junjie, Van Dael and Vangeel, Jacobson's Free Length Theory, Impedance Dependence Relation and Schaaf's Collision Factor Theory together with experimental values for the ternary mixture of tetrahydrofuran $\left(\mathrm{x}_{1}\right)+$ heptane $\left(\mathrm{x}_{2}\right)+\operatorname{decane}\left(\mathrm{x}_{3}\right)$ at the temperatures $303 \mathrm{~K}$, 
$308 \mathrm{~K}$ and $313 \mathrm{~K}$ are summarised in Table 1. The percentage deviation, Average Percentage Error (APE) and of sound velocity using the above theory and molecular interaction term $\alpha$ for the mixtures studied are given in Table 2 .

Table 1. Experimental and theoretical values of velocities in tetrahydrofuran $\left(\mathrm{x}_{1}\right)+$ heptane $\left(\mathrm{x}_{2}\right)+$ decane $\left(\mathrm{x}_{3}\right)\left(\mathrm{x}_{2} / \mathrm{x}_{3}=3: 1\right)$ at 303,308 and $313 \mathrm{~K}$

\begin{tabular}{|c|c|c|c|c|c|c|c|}
\hline $\begin{array}{c}\text { Mole Fraction } \\
\mathrm{X}_{1} \\
\end{array}$ & $\begin{array}{l}U_{\text {EXP }} \\
m^{-1}\end{array}$ & $\begin{array}{l}U_{\mathrm{NR}} \\
\mathrm{ms}^{-1} \\
\end{array}$ & $\begin{array}{l}\mathrm{U}_{\mathrm{JM}} \\
\mathrm{ms}^{-1}\end{array}$ & $\begin{array}{l}\mathrm{U}_{\mathrm{IMR}} \\
\mathrm{ms}^{-1}\end{array}$ & $\begin{array}{l}U_{\text {FLT }} \\
\mathrm{ms}^{-1}\end{array}$ & $\begin{array}{l}\mathrm{U}_{\mathrm{CFT}} \\
\mathrm{ms}^{-1}\end{array}$ & $\begin{array}{l}U_{\text {IDR }} \\
\mathrm{ms}^{-1} \\
\end{array}$ \\
\hline \multicolumn{8}{|c|}{$303 \mathrm{~K}$} \\
\hline 0.0 & 1157.0 & 1130.0 & 1126.2 & 1105.6 & 1155.4 & 1101.0 & 1126.8 \\
\hline 0.1 & 1189.0 & 1136.1 & 1128.6 & 1114.2 & 1170.8 & 1098.8 & 1142.4 \\
\hline 0.2 & 1171.1 & 1143.0 & 1131.8 & 1123.9 & 1143.2 & 1090.4 & 1157.1 \\
\hline 0.3 & 1146.0 & 1150.6 & 1135.8 & 1134.7 & 1157.2 & 1095.0 & 1171.1 \\
\hline 0.4 & 1142.2 & 1159.3 & 1141.1 & 1146.7 & 1107.7 & 1079.3 & 1184.2 \\
\hline 0.5 & 1130.0 & 1169.2 & 1148.1 & 1159.9 & 1091.6 & 1074.0 & 1196.7 \\
\hline \multicolumn{8}{|c|}{$308 \mathrm{~K}$} \\
\hline 0.0 & 1103.0 & 1082.6 & 1078.1 & 1056.8 & 1138.4 & 1070.1 & 1079.0 \\
\hline 0.1 & 1143.7 & 1088.5 & 1080.3 & 1065.4 & 1114.6 & 1060.8 & 1094.2 \\
\hline 0.2 & 1134.1 & 1095.0 & 1083.1 & 1075.0 & 1104.7 & 1056.1 & 1108.5 \\
\hline 0.3 & 1123.8 & 1102.3 & 1086.8 & 1085.6 & 1103.7 & 1054.2 & 1122.0 \\
\hline 0.4 & 1116.2 & 1110.6 & 1091.8 & 1097.3 & 1070.8 & 1040.8 & 1134.7 \\
\hline 0.5 & 1106.7 & 1120.0 & 1098.3 & 1110.2 & 1049.7 & 1031.2 & 1146.7 \\
\hline \multicolumn{8}{|c|}{$313 \mathrm{~K}$} \\
\hline 0.0 & 1048.0 & 1040.0 & 1035.8 & 1015.5 & 1098.2 & 1042.1 & 1036.5 \\
\hline 0.1 & 1079.6 & 1045.6 & 1037.8 & 1023.7 & 1090.7 & 1037.0 & 1051.2 \\
\hline 0.2 & 1068.0 & 1051.8 & 1040.3 & 1032.8 & 1082.7 & 1031.5 & 1065.0 \\
\hline 0.3 & 1062.5 & 1058.8 & 1043.8 & 1042.9 & 1065.3 & 1022.0 & 1077.9 \\
\hline 0.4 & 1055.2 & 1066.7 & 1048.3 & 1054.2 & 1035.0 & 1007.2 & 1090.1 \\
\hline 0.5 & 1045.5 & 1075.7 & 1054.5 & 1066.5 & 1024.3 & 999.6 & 1101.6 \\
\hline
\end{tabular}

It is assumed that all the molecules are spherical in shape, which is not true every time. In Nomoto's theory, it is supposed that the volume does not change on mixing. Therefore, no interactions between the components of liquid mixtures have been taken into account. The assumption for the ideal mixing relation is that the ratio of specific heats of ideal mixtures and the volumes are also equal. Again no molecular interaction is taken into account. Similarly as per the assumption for the collision factor theory, the molecules are treated as real nonelastic substances, which is not really the case. But on mixing two liquids, the interaction between the molecules of the two liquids takes place because of presence of various types of forces such as dispersion forces, charge transfer, hydrogen bonding, dipoledipole and dipole-induced dipole interactions. Thus the observed deviation of theoretical values of velocity from the experimental values shows that the molecular interaction is taking place between the unlike molecules in the liquid mixtures.

In general, the predictive abilities of various ultrasonic theories depend upon the strength of interactions that exist in a ternary system. In case strong interactions exist between the molecules of the mixtures, there is much deviation in theoretical prediction of velocity than the molecules of the mixture where less interaction are present.

A general survey of Table 2 shows that $\alpha$ is positive. The positive values of $\alpha$ in the system at all the temperatures clearly indicate the existence of strong tendency for the formation 
of association in mixture through hydrogen bonded complexes ${ }^{14}$. As is evident from the APE values (Table 2), the best results are obtained by NR followed by the results obtained from other theories.

Table 2. Percentage deviation, Average Percentage Error and molecular interaction parameter values for the system tetrahydrofuran $\left(\mathrm{x}_{1}\right)+$ heptane $\left(\mathrm{x}_{2}\right)+$ decane $\left(\mathrm{x}_{3}\right)\left(\mathrm{x}_{2} / \mathrm{x}_{3}=3: 1\right)$ at 303, 308 and $313 \mathrm{~K}$

\begin{tabular}{|c|c|c|c|c|c|c|c|}
\hline $\begin{array}{c}\text { Mole } \\
\text { Fraction } \mathrm{X}_{1} \\
\end{array}$ & $\% U_{N R}$ & \% U JM & $\% U_{\text {IMR }}$ & $\% U_{\text {FLT }}$ & $\begin{array}{c}\% \mathrm{U} \\
\mathrm{CFT}\end{array}$ & $\% U_{\text {IDR }}$ & $\mathrm{a}$ \\
\hline \multicolumn{8}{|c|}{$303 \mathrm{~K}$} \\
\hline 0.0 & 2.33566 & 2.66141 & 4.44535 & 0.13698 & 4.83933 & 2.61384 & 0.0952 \\
\hline 0.1 & 4.44805 & 5.07675 & 6.28817 & 1.53139 & 7.58935 & 3.91943 & 0.1387 \\
\hline 0.2 & 2.40324 & 3.35791 & 4.02871 & 2.38409 & 6.89078 & 1.19231 & 0.0857 \\
\hline 0.3 & -0.40440 & 0.88639 & 0.98611 & -0.97554 & 4.45248 & -2.18588 & 0.0200 \\
\hline 0.4 & -1.49816 & 0.09241 & -0.39029 & 3.02477 & 5.50747 & -3.67760 & -0.0078 \\
\hline 0.5 & -3.46873 & -1.60387 & -2.64567 & 3.39526 & 4.95984 & -5.89939 & -0.0509 \\
\hline APE & 0.63594 & 1.74517 & 2.11873 & 1.58282 & 5.70654 & -0.67288 & - \\
\hline \multicolumn{8}{|c|}{$308 \mathrm{~K}$} \\
\hline 0.0 & 1.84790 & 2.25893 & 4.18690 & -3.21007 & 2.98155 & 2.17453 & 0.0893 \\
\hline 0.1 & 4.83043 & 5.54763 & 6.84544 & 2.54246 & 7.24864 & 4.32684 & 0.1524 \\
\hline 0.2 & 3.45033 & 4.49721 & 5.21406 & 2.59327 & 6.88017 & 2.25694 & 0.1130 \\
\hline 0.3 & 1.91409 & 3.28922 & 3.40220 & 1.78634 & 6.19120 & 0.16334 & 0.0717 \\
\hline 0.4 & 0.50423 & 2.18867 & 1.69431 & 4.06681 & 6.75138 & -1.65430 & 0.0348 \\
\hline 0.5 & -1.20356 & 0.75578 & -0.31901 & 5.14633 & 6.82246 & -3.61156 & -0.0063 \\
\hline APE & 1.89057 & 3.08957 & 3.50398 & 2.15419 & 6.14590 & 0.60930 & - \\
\hline \multicolumn{8}{|c|}{$313 \mathrm{~K}$} \\
\hline 0.0 & 0.76221 & 1.16157 & 3.10236 & -4.78703 & 0.55941 & 1.09283 & 0.0651 \\
\hline 0.1 & 3.15203 & 3.87554 & 5.17928 & -1.02447 & 3.94565 & 2.63043 & 0.1122 \\
\hline 0.2 & 1.51872 & 2.59020 & 3.29463 & -1.37364 & 3.42205 & 0.28490 & 0.0693 \\
\hline 0.3 & 0.35187 & 1.76317 & 1.84071 & -0.26085 & 3.80839 & -1.44881 & 0.0379 \\
\hline 0.4 & -1.08683 & 0.64988 & 0.09934 & 1.91108 & 4.54607 & -3.30572 & 0.0020 \\
\hline 0.5 & -2.88830 & -0.85983 & -2.01188 & 2.02538 & 4.38632 & -5.36446 & -0.0391 \\
\hline APE & 0.30162 & 1.53009 & 1.91741 & -0.58492 & 3.44465 & -1.01847 & - \\
\hline
\end{tabular}

Keeping in view of the aforementioned system, it can be thought that positive deviations in velocity are a result of (a) molecular association and (b) complex formation whereas negative deviations in velocity are due to molecular dissociation of associated species as a result of addition of inner solvent or an active solvent. The actual sign and magnitude of deviations depend upon relative strength of two opposite effects. The lack of smoothness in deviations is due to the interaction between the component molecules ${ }^{15}$.

The deviations between theoretical and experimental values of ultrasonic velocities decrease with increase of temperature due to breaking of hetero and homo molecular clusters at higher temperatures ${ }^{16}$. On increasing the temperature, the ultrasonic velocity values decrease in the ternary liquid mixture. This is probably due to the fact that the thermal energy activates the molecule, which would increase the rate of association of unlike molecules. Based on the theoretical values of ultrasonic velocity; it is worthwhile to state that NR method yield the best result at all temperatures. The magnitude of APE is in order: $\mathrm{NR}<\mathrm{IDR}<\mathrm{FLT}<\mathrm{JM}<\mathrm{IMR}<\mathrm{CFT}$. 


\section{Conclusion}

It may be concluded that out of six theories and relations discussed above, the Nomoto's mixing relation, Impedance dependence provided good results. Thus the linearity of molar sound velocity and additivity of molar volumes, as suggested by Nomoto and Impedance relation, in deriving the empirical relations have been truly observed in the studied ternary liquid mixtures.

\section{Acknowledgement}

I am very much thankful to Dr.R.Palani, Associate Prof. of Physics, Annamalai University and Dr.G.Srinivasan, Asst. Prof. of Physics, Achariya College of Engineering Technology, Puducherry, for their continuous support and encouragement.

\section{References}

1. Rama Rao G V, Viswanatha Sarma A, Siva Rama Krishna J and Rambabu C, Indian J Pure Appl Phys., 2005, 43(5), 345-354.

2. $\quad$ Balaja S and Oza S, J Pure Appl Ultrasonics, 2002, 24, 97.

3. Ali A. Yasmin A and Nain A K, Indian J Pure Appl Phys., 2002, 40(5),.315-322.

4. Rastogi M Awasthi A Gupta M and Shukla J P, Indian J Pure Appl Phys., 2002, 40(4), 256-263.

5. $\quad$ Ali A and Nain A K and Hyder S, J Pure Appl Ultrasonics, 2001, 23, 73-79.

6. Nomoto O, J Phys Soc Japan, 1958, 13, 1528-1532; DOI:10.1143/JPSJ.13.1528

7. Junjie Z, J University of Science and Technology of China, 1984, 14, 298.

8. VanDael W and Vangeel, in Proceedings of the International Conference on Calorimetry and Thermodynamics, p.555, Warsaw, Poland, 1955.

9. Jacobson B, Acta Chem Scand, Denmark, 1952, 6, 1485-1487.

10. Schaaf'sW, Acoustica, 1975, 33, 272-276.

11. Kalidass M and Srinivasamoorthy R, J Pure Appl Ultrasonics, 1997, 19, 9-15.

12. Palaniappan P L R M, Pichaimuthu A and Kannappan A N, Indian J Physics, 1997, 71A(5), 175-179.

13. Suhasini Ernest and Kavitha P, International Journal of Chemical, Environmental and Pharmaceutical Research., 2011, 2(2-3), 92-95.

14. Shanthi N, Sabarathinam P L, Emayavaramban M, Gopi C and Manivannan C, $J$ Chem., 2010, 7(2), 648-654; DOI:10.1155/2010/487874

15. Pandey J D, Singh A K, and Dey R, Pramana, 2005, 64(1), 135-139.

16. Begum Z, Sandhya Sri P B and Rambabu C, ISRN Physical Chemistry, 2012, 2012, Article ID 943429, 12 pages; DOI:10.5402/2012/943429 